\title{
Civilisations
}

Revue internationale d'anthropologie et de sciences

humaines

59-1 | 2010

Sexualités : apprentissage et performance

\section{Sexualités et prévention dans les romans contemporains sur le VIH/sida}

Une source d'apprentissage?

Joseph Josy Lévy et Lucie Quevillon

\section{OpenEdition}

Journals

Édition électronique

URL : http://journals.openedition.org/civilisations/2243

DOI : $10.4000 /$ civilisations.2243

ISSN : 2032-0442

Éditeur

Institut de sociologie de l'Université Libre de Bruxelles

\section{Édition imprimée}

Date de publication : 28 juin 2010

Pagination : 59-88

ISBN : 2-87263-029-5

ISSN : 0009-8140

Référence électronique

Joseph Josy Lévy et Lucie Quevillon, «Sexualités et prévention dans les romans contemporains sur le VIH/sida », Civilisations [En ligne], 59-1 | 2010, mis en ligne le 29 juin 2013, consulté le 30 avril 2019.

URL : http://journals.openedition.org/civilisations/2243; DOI : 10.4000/civilisations.2243 


\title{
Sexualités et prévention \\ dans les romans contemporains sur le VIH/sida \\ Une source d'apprentissage?
}

\author{
Joseph Josy LÉVY et Lucie QUEVILLON
}

Résumé : Les romans peuvent contribuer à l'apprentissage de la sexualité et, à travers la fiction, nourrir les scénarios érotiques. Dans le monde contemporain, l'épidémie du VIH/sida a inspiré de nombreux romans qui mettent en relief les enjeux entourant l'expression de la sexualité et la prévention. L'analyse d'un corpus de romans, qui abordent surtout l'homosexualité, parus après 1996 en langues anglaise et française, a permis de cerner les représentations de l'espace sexuel, les scénarios entourant les choix relationnels et le registre des comportements érotiques et préventifs. Elle met aussi en relief des réflexions éthiques sur l'érotisme, sur la question des risques, sur la prévention et ses limites, qui peuvent contribuer à enrichir le champ de l'anthropologie de la sexualité et ses rapports à la maladie.

Mots-clés : romans, VIH/sida, sexualité, scénarios sexuels, littérature.

\begin{abstract}
Novels can contribute to the learning of sexuality, in feeding erotic scenarios through fiction. In the contemporary world, the HIV epidemic inspired numerous novels that highlight issues of the expression of sexuality and its prevention. The analysis of a corpus of novels dealing mainly with homosexuality, published in English or French after 1996, allowed us to bring to light representations of the sexual space, as well as scenarios leading to the choice of partners, and diverse erotic and preventive practices. In the novels, our contribution also highlight ethical reflections on eroticism, on prevention as well as on its limits, which can contribute to enrich the field of the anthropology of sexuality and of its relations to health issues.
\end{abstract}

Key words: novels, HIV/AIDS, sexuality, sexual scenarios, literature. 


\section{Introduction}

L

a transmission, définie comme «l'ensemble des processus par lesquels un groupe humain assure sa continuité dans le temps, à travers la succession des générations » (Hervieu-Léger 1997 : 131), constitue l'une des problématiques essentielles dans le champ de la sociologie et de l'anthropologie. C'est en effet à travers les modes de socialisation, portés par différents agents et institutions, que se transmet l'héritage culturel dans ses différentes dimensions : abstraites (valeurs, croyances, normes et savoirs) et concrètes (comportements, gestes, savoir-faire et pratiques) (Bruner 1956 ; Pelissier 1991 ; Trueba 1993 ; Ohmagari et Berkes 2004). Les processus de transmission varient selon les contextes socioculturels, les critères de classe, d'âge et de genre, mais aussi en fonction des champs d'activités. La transmission culturelle des valeurs et des conduites sexuelles constitue un domaine particulièrement sensible à cause de la grande importance reconnue transculturellement à la sexualité (Reiss 1986) et des liens qu'elle entretient avec d'autres composantes de la vie sociale (rapports de parenté, systèmes d'alliance, interdits, comportements reproductifs, etc.).

Plusieurs stratégies de socialisation sexuelle sont ainsi discernables. Dans nombre de sociétés, l'apprentissage des normes et des pratiques sexuelles est limité et il s'effectue de façon détournée et souvent implicite, à cause des interdits et des conduites d'évitement entourant cette sphère d'activité. C'est, par exemple, le cas de la société irlandaise d'Inis Beag (Messenger 1971) où l'information sexuelle en milieu familial était quasi absente et dépendait des connaissances transmises par le groupe des pairs et de l'observation des animaux. Ailleurs, l'instruction peut aussi faire appel aux ressources orales. Par exemple, les proverbes, chez les Tamouls, contribuent à la structuration des normes sexuelles en insistant sur " la noblesse du désir sexuel en même temps qu'ils vantent la continence » (Bourdier 2001 : 198) ; de même, les contes dans certaines sociétés d'Afrique de l'Ouest (Lallemand 1985), les chansons érotiques, ou même pornographiques, au Japon (Maring et Maring 1997), orientent l'éducation à la sexualité.

Ces ressources servent à définir les normes de conduites sexuelles, en mettant en évidence, d'une part, les dilemmes et les tensions associées à la sexualité, et en offrant, d'autre part, des intrigues et des exemples qui peuvent alimenter l'imaginaire sexuel des auditeurs. Ces modes d'éducation orale peuvent être complétés, dans certains contextes, par un apprentissage des techniques du corps entourant la sexualité (postures, mouvements, réponse sexuelle, etc.) qui servent à ancrer les modèles valorisés, formes de conditionnement dont Mauss (1936) avait souligné l'importance'. Ainsi, dans plusieurs rituels d'initiation africains, comme c'est le cas chez les Luvale (White 1953), les Vendas (Blacking 1985), les Ambos (Stefaniszyn 1964) ou les Zoulous (Krige 1968), en plus de l'apprentissage des identités de genre et des rôles sexuels, vient s'ajouter celui des modalités corporelles touchant la sphère érotique valorisées par les groupes socioculturels. De telles initiations féminines peuvent comprendre une éducation sexuelle sur les méthodes contraceptives

1. D'après C. Rivière, l'apprentissage des rituels du corps a pour fonction, entre autres, d'inhiber « les conduites qui ne répondraient pas au code collectif de l'échange fondé sur la présentation d'un corps socialisé et sexué » (1995: 147). 
et abortives de même que sur l'apprentissage des techniques sexuelles (exercices visant à intégrer les mouvements des hanches et du bassin) pour augmenter le plaisir lors de la relation coïtale ${ }^{2}$. Dans d'autres contextes socioculturels, sans s'inscrire dans des rituels d'initiation, une éducation sexocorporelle explicite est aussi présente comme c'est le cas, par exemple, à Mangaia (Marshall 1971) et aux îles Marquises (Suggs 1966)³. Dans la région des Grands Lacs africains (Kashamura 1973), les jeunes filles s'initient entre elles à la vie sexuelle et leurs activités portent sur les techniques qui permettent de modifier les zones génitales, et sur les exercices concourant à l'intégration de la réponse orgastique (caresses du clitoris et des lèvres), accompagnées de techniques mentales visant au développement de l'imaginaire érotique ${ }^{4}$. Ces modalités éducatives basées surtout sur l'apprentissage corporel semblent constituer un cas particulier de la procédure liée à la production de la « vérité du sexe ». Foucault définit de concept de « vérité du sexe » comme celle de l'ars erotica où « la vérité est extraite du plaisir lui-même, pris comme pratique et recueilli comme expérience » (1976 : 77). Par ailleurs, d'autres sociétés et d'autres civilisations encore (en Chine, au Japon, en Inde ou dans le monde arabo-musulman) ont développé des pratiques mystico-érotiques faisant intervenir un savoir secret transmis de maître à disciple (voir Evola 1968).

Les innovations liées à l'écriture et l'imprimerie (xylographie en Asie ou typographie en Occident) vont transformer les modalités d'éducation à la sexualité en contribuant à la diffusion de nombreux ouvrages érotiques qui vont permettre une plus grande autonomie dans les modes d'apprentissage en réduisant, en partie, le contrôle direct des instances éducatives sur les apprenants. Même si les ouvrages continuent à véhiculer les normes sexuelles dominantes et propres à chaque groupe socioculturel, ces instances ne peuvent les imposer rigoureusement et, de ce fait, les lecteurs, à travers les textes et les images qui accompagnent ces manuels, sont à même de développer leurs compétences érotiques et de les évaluer de façon plus individuelle, s'émancipant du même coup de rapports fortement personnalisés. Comme l'a montré Goody (2003), les manuels érotiques vont prendre une place importante dans l'instruction sexuelle. En Inde, dans le Kama Sutra :

[...] un genre de vade-mecum du sexe pour les jeunes mariés, [...] les renseignements qu'on y trouve ne sont pas une description générale des comportements, mais un ensemble d'instructions, exposant par exemple un savoir nouveau ou restreint

2. L'exemple des transformations du rite d'initiation chez les Luvale (White 1953) illustre l'importance de la dimension érotique : bien que ce rite ait perdu la plupart de ses fonctions, il a gardé celle qui touche la sexualité, permettant ainsi aux femmes de développer leur compétence érotique.

3. À Mangaia (Marshall 1971), le cunnilingus, les méthodes de contrôle de l'éjaculation et l'atteinte de l'orgasme simultané sont enseignés aux garçons qui, une fois cette période d'instruction achevée, passent à des exercices pratiques avec une femme expérimentée. De la même façon, les jeunes filles apprennent à développer la réponse orgastique sous la conduite des « good-men » qui l'initient à cette fin. L'apprentissage érotique peut aussi se faire d'une manière informelle par la transmission d'informations, de discussions et d'exercices dans le groupe des pairs. Chez les Marquisiens (Suggs 1966), une jeune monitrice montre les positions coïtales ainsi que les mouvements du bassin en adoptant successivement le rôle du garçon et de la fille.

4. Kashamura (1973) précise que celles chez qui l'excitation sexuelle peut être atteinte par la seule imagination sont considérées comme des expertes. Les garçons impubères participent à ces activités comme partenaires et comme apprentis, ce qui permet une co-éducation sexuelle. 
concernant les positions sexuelles et contribuant ainsi à la différentiation de souscultures dans la société indienne. (Goody $2003: 231)^{5}$

Dans le monde chinois (Goody 2003 : 250-251), les manuels de la chambre à coucher jouent aussi un rôle important et sont diffusés dans les classes privilégiées ${ }^{6}$. Ces types d'ouvrages se retrouvent dans le contexte japonais avec les manuels de l'oreiller (Soulié 1993) qui comprennent des textes et des images érotiques (shunga) sur les positions à privilégier. Dans le monde islamique, l'érotologie élaborée, qui comprend plusieurs ouvrages dont l'un des plus connus est sans doute le Jardin parfumé du cheikh Nefzaoui, rédigé au $16^{\mathrm{e}}$ siècle, explore aussi le champ de l'érotisme et propose des modalités d'amplification du plaisir ${ }^{7}$ (Bouhdiba 2003).

Les manuels ne sont pas étrangers à la civilisation occidentale et, à partir de l'Antiquité, plusieurs de ces ouvrages ont circulé, exposant des « listes de positions recommandées dans les rapports hétérosexuels et bien d'autres précisions encore »(Goody 2003 : 242). Avec l'ascendance du christianisme, dont l'idéologie tendait à restreindre l'exploration de l'érotisme (Ranke-Heinemann 1990), ce type de littérature fut soumis à la censure ecclésiastique. Les manuels continuèrent cependant à circuler sous le manteau, puis au grand jour, proposant des normes plus ou moins permissives, selon les auteurs et les périodes, qui orientèrent les modalités de l'expression érotique (Melody et Peterson 1999).

Parallèlement à cette production, la littérature romanesque, amoureuse et érotique, est venue diversifier les modèles érotiques en privilégiant, à travers un traitement rhétorique, la présentation de personnages, d'intrigues, de sentiments et de conduites qui reflétaient les normes culturelles dominantes, ou s'en éloignaient en traitant de pratiques transgressives que l'on retrouve dans les canons littéraires chinois, pouvant aller jusqu'à des « descriptions

5. Rédigé, selon la tradition, par Vatsyayana, un brahmane du $3^{\mathrm{e}}$ siècle de l'ère chrétienne, cet ouvrage, dont le titre signifie « Aphorismes sur l'amour », insiste sur la notion de kama ou plaisir, ce qui exige un apprentissage du raffinement des sens afin d'amplifier l'excitation et la modulation de la réponse érotique. Il présente donc sous une forme condensée des conseils quant aux approches de séduction, au choix des partenaires et à la typologie des actes érotiques. À part la typologie des femmes et des hommes classés en fonction de la dimension des organes sexuels, l'essentiel de l'ouvrage porte sur la description et la classification des actes érotiques : baisers en fonction des zones érogènes, du type de toucher, de pénétration, de frottement ou de pression. Différentes positions sont ainsi proposées, tout comme les signaux éroticocorporels qui indiquent jouissance et satisfaction.

6. Ces manuels, diffusés pendant la période de l'apogée de l'Empire, entre 590 et 1279 de l'ère chrétienne, avant que la Chine ne devienne beaucoup plus prude, règlent les arts de la chambre à coucher. Ils exposent les approches érotiques conformes aux perspectives taoïstes qui considèrent le corps humain comme un microcosme de l'univers, parcouru par des énergies fondamentales, le yin et le yang, forces à la fois opposées et complémentaires dont l'harmonie est nécessaire pour le maintien du bien-être et de la longévité, ou pour atteindre l'immortalité (Van Gulik 1977). L'art érotique met ainsi en scène ce que la poétique érotique chinoise nomme «Les jeux des nuages et de la pluie », pour illustrer, de façon sensible et tendre, les différents aspects de l'activité érotique en insistant sur le contexte, les signaux corporels indiquant le désir ou l'orgasme, les postures sexuelles aux noms poétiques, les réactions affectives des partenaires, les types de poussées et leurs rythmes.

7. Ce petit ouvrage, rédigé pour un bey de Tunis, présente les modèles d'homme et de femme idéaux sur le plan érotique et prodigue de nombreux conseils sur l'initiation de la relation sexuelle, les préliminaires (baisers, succion, caresses, morsures), les positions sexuelles, la réponse orgastique, de même que sur le traitement des dysfonctions érotiques. Il s'inscrit dans une vaste littérature érotique qui fait appel à plusieurs formes, de la poésie aux contes dont les plus connus sont Les Mille et Une nuits. 
paillardes » (Goody 2003 : 251) et dans la littérature japonaise et occidentale (Évrard 2003 ; Alexandrian 1995 ; Brulotte 1998). La littérature romanesque érotique a ainsi constitué un nouveau développement dans les modalités de transmission des savoirs et savoir-faire sexuels en insistant sur les fonctions liées à l'imaginaire érotique qu'elle contribue à structurer et à enrichir en nourrissant les fantasmes ${ }^{8}$.

La dimension pédagogique est aussi soulignée par Turner (2003) qui, dans son étude sur les textes érotiques italiens, français et anglais qui s'inscrivent dans la tradition libertine, montre leur contribution à la pédagogie sexuelle orientée vers les jeunes femmes et dégage les enjeux qu'elle soulève. Cette perspective est reprise dans des travaux sur les romans érotiques ultérieurs (Kraakman 1994 ; Hubier 2007). La construction de soi pourrait ainsi être influencée par la lecture. Pour d'autres auteurs, cependant, les répercussions du roman et de la fiction en général dépassent le plan de l'imaginaire et orientent la construction identitaire suite aux mécanismes d'identification (De Singly 1993). Bourneuf (2007) insiste aussi sur ces fonctions du livre qui « suscite émotions et sentiments, active, relance la réflexion, tout ensemble nourrit l'imaginaire et nous rapproche de la vie palpable, décrit les existences réelles et fictives, propose des modes d'être possibles, trace des voies pour notre évolution individuelle et collective " (Bourneuf 2007 : 10). Le rapport à la fiction s'est aussi diversifié avec le développement des productions télévisuelles, des films, des reportages et des informations disponibles sur internet qui présentent des modèles de conduites sexuelles. À partir de cette constatation, Weitman (1999) commente :

[la société contemporaine] est caractérisée par la tendance des individus [...] à être guidés dans la vie réelle par des dispositions d'habitus acquises au cours d'une immersion prolongée dans le monde imaginaire de la fiction, et moins par les dispositions acquises au cours de l'adaptation à la vie et aux personnes réelles. (Weitman 1999 : 73-74)

Ces recherches suggèrent donc que la production romanesque constitue un matériau intéressant d'un point de vue anthropologique en permettant d'accéder à un ensemble de représentations sociales (Jodelet 1989) ${ }^{9}$ sur la sexualité et au contenu des scénarios sexuels (Gagnon 2008). L'analyse des romans peut être particulièrement intéressante pour comprendre le retentissement d'évènements socioculturels majeurs sur la sexualité, comme ce fut le cas avec l'épidémie du VIH/sida qui a modifié les représentations de la sexualité et de ses risques, de même que les pratiques (Parker 2001). Dès les années 1980, soit les premières années qui ont vu l'apparition de cette épidémie, on voit en effet se mettre en place une création littéraire et artistique de plus en plus diversifiée (romans, poésie, théâtre, cinéma) qui explore les différentes facettes de l'épidémie. Sa construction et sa traduction

8. Comme le note Alexandrian (1995: 389) : «La plupart des auteurs [de cette littérature] expriment leurs fantasmes plutôt que des expériences réelles, et ces fantasmes exagèrent ou déforment les véritables possibilités du sexe. Elle [cette littérature] n'en est pas moins intéressante et authentique, puisqu'une partie de la sexualité humaine tend à s'assouvir dans l'imaginaire. " Cette fonction de la littérature érotique est aujourd'hui utilisée dans les thérapies sexuelles qui ont recours à ce type de textes pour enrichir la vie fantasmatique des patients et nourrir le désir par l'évocation de scènes érotiques (Hubin, De Sutter et Reynaert 2008).

9. Le matériau littéraire a été utilisé pour l'étude de la maladie (Laplantine 1986), la magie (Garnier 1999) ou l'excision en Afrique (Zabus 2007). Dans le champ de la sexualité, l'analyse des romans africains a mis en relief les modalités du rapport au pouvoir et à la sexualité (Cornaton 1990). 
dans l'imaginaire romanesque ont fait aussi l'objet de plusieurs recherches (Murphy et Poirier 1993 ; Volet, Jaccomard et Winn 2002).

Au plan anthropologique, la recension d'un premier corpus de romans publiés jusqu'en 1994, par des auteurs anglo-saxons et français, et qui traitaient du thème du VIH/sida, soit une quinzaine de romans et trois recueils de nouvelles, a été réalisée par Lévy et Nouss (1994). Cette étude a cerné les représentations et l'expérience des maladies liées au VIH/ sida, son encadrement bio-médical, le retentissement de l'épidémie sur le rapport à la mort, le deuil et les rites, ainsi que sur les constructions de l'identité sexuelle, en particulier homosexuelle, et l'expression de la sexualité confrontée aux enjeux de la prévention et du risque. Dans cet article, nous poursuivons cette analyse en dégageant les scénarios sexuels présents dans des romans contemporains parus après $1996^{10}$ ayant pour thématique le VIH/ sida et la sexualité, et nous envisageons successivement les représentations imaginaires des lieux, des relations interpersonnelles, des pratiques sexuelles, des risques et des stratégies préventives ${ }^{11}$.

\section{L'imaginaire des lieux}

La lecture des romans et leur comparaison met en relief l'importance de l'espace géographique et des lieux dans lesquels évoluent les personnages à des fins de loisirs, de rencontres amoureuses et sexuelles. L'imaginaire spatial peut renvoyer à des destinations exotiques comme Madagascar, Honolulu ou Haïti, mais c'est surtout le monde européen et américain, et leurs villes, qui retient l'attention des auteurs, complétant, en cela, l'importance de la ville présente dans l'imaginaire d'autres corpus littéraires (Nicolas 2002), mais en en précisant la spatialisation sexuelle. Pour rendre compte de cette interface, Bech (1999) a proposé le concept de Citysex qui renvoie non seulement à la sexualisation de la ville contemporaine mais aussi à la conception de la sexualité moderne comme essentiellement urbaine. Dans cette perspective, l'espace urbain moderne est caractérisé par la présence d'une foule composée d'étrangers en contact les uns avec les autres et par l'existence d'espaces publics et privés, mais aussi télémédiatisés. Dans ce contexte, même si plusieurs figures prototypiques sont présentes (poètes, flâneurs, prostituées, etc.), l'homosexuel masculin, selon Bech, constitue la figure urbaine par excellence et il occupe pleinement cet espace, contrairement aux autres prototypes.

L'univers urbain décrit dans les romans analysés semble confirmer ces hypothèses en présentant les personnages, surtout d'orientation homosexuelle, et les intrigues dans le contexte des grandes villes cosmopolites dans lesquelles on retrouve des populations homosexuelles importantes (Sibalis 2004 ; Collins 2007). Cette géographie romanesque,

10. Cette analyse se base sur un corpus préliminaire de 16 romans qui soulèvent la thématique du VIH/sida et de la sexualité. Ce corpus ne prétend pas à une exhaustivité ou à une représentativité. Parus entre 1997 et 2007, les auteurs de ces romans sont des hommes, et les textes, pour la plupart, sont écrits à la première personne. La grande majorité des personnages principaux sont des hommes, d'orientation surtout homosexuelle.

11. Les textes ont été analysés et codifiés en fonction de catégories touchant le VIH/sida (origine, représentations, expérience, encadrement bio-médical, traitements pharmacologiques, alternatifs et complémentaires, stigmatisation et discrimination, interprétation de la maladie), la mort (représentations de la mort, croyances, expérience de deuil, rituels mortuaires) et les scénarios sexuels (espaces sexualisés, constructions de l'identité sexuelle, affects et les pratiques, risques et stratégies de prévention). Nous ne retenons ici, faute d'espace, que quelques dimensions des scénarios. 
qui ne se fonde par toujours sur des descriptions détaillées, peut faire état de localisations toponymiques ou anonymes et insiste en particulier sur l'espace public plutôt que privé, signifiant ainsi l'effacement de la dimension clandestine de la présence homosexuelle qui avait cours dans le passé (Demczuk et Remiggi 1998 ; Deligne, Gabiam, Van Criekingen et Decroly 2006).

À part la mention de la ville de Port-au-Prince en Haïti, ce sont surtout les références aux villes européennes (Amsterdam, Barcelone, Lyon, Madrid, Nancy, Paris, Valence) ou nord-américaines (Los Angeles, Montréal, New York, Québec, San Francisco, Toronto) associées à la vie nocturne et homosexuelle, à la présence de grandes communautés gaies et à la diversité des espaces de convivialité, qui dominent et que parcourent les narrateurs et les personnages dans leur vie quotidienne. Des rues (Christopher Street ou Amsterdam Avenue à New York, boulevard Saint-Laurent ou rue Laurier à Montréal) de même que des quartiers où l'on retrouve une forte présence homosexuelle (le Marais, West Village, le Village et le quartier du Plateau montréalais) sont ainsi spécifiquement mentionnés. Dans le cas de Paris, le quartier du Marais constitue un point d'attraction lié à l'exubérance de ses activités, à l'instar des parcs d'amusement modernes comme Dysneyland ${ }^{12}$ New York attire pour ses possibilités multiples de rencontres sexuelles aventureuses ${ }^{13}$ La géographie sociale de l'univers gai est aussi esquissée, mettant en évidence la diversité des styles de vie de couple et la répartition spatiale en fonction de critères de distinction : les pantouflards se retrouvent à Brooklyn Heights, les chics dans l'Upper East Side et les intellectuels dans l'Ouest de la ville ${ }^{14}$.

Les romans soulignent également les attraits de la vie urbaine, pour de jeunes homosexuels dont la vie ne peut être circonscrite aux limites d'une petite ville qui contraint leurs possibilités d'autonomie, d'anonymat, d'expérimentation et de liberté ${ }^{15}$. Ces représentations stéréotypées et idéalistes des avantages de la ville rejoignent les constatations de Bech (1999) sur la sexualité urbaine qui se fonderait sur « l'excitation, l'offre, les occasions et la liberté », mais aussi sur la « liberté potentielle intérieure « d'être soi-même » en lien avec l'anonymat et la non-implication des relations urbaines » (Bech 1999 : 219). Il est à noter que cette analyse sous-estime les problèmes de solitude, d'aliénation, de violence et d'exploitation sexuelle que l'on retrouve dans les milieux urbains. À la liberté s'ajoute l'effervescence de

12. «Le Marais, c'est Disneyland. X : [Qu'est-ce que ce « X »? Où commence le guillemet d'ouverture ?] Pourquoi Disneyland? Les gens, ils viennent là parce que c'est l'endroit le plus civilisé à des centaines et des centaines de kilomètres à la ronde. Ils sont attirés et puis c'est vivant, alors que partout ailleurs, c'est mort. » (Dustan, Génie divin, 2001, p. 84).

13. «[...] en réalité j'étais trop accroché au genre d'aventure sexuelle que seul New York pouvait fournir. » (White, La symphonie des adieux, 1998, p. 218).

14. «Les couples gays étaient généralement relégués à Brooklyn Heights (s’ils étaient popotes) ou dans le Upper East Side (s'ils étaient chics) ou à l'Ouest (s'ils étaient intellos) - en tout cas hors de vue de ces Villageois intrépides [...]» (White, La symphonie des adieux, 1998, p. 254-255).

15. «Montréal, j’ai besoin de Montréal. Besoin d'espace, de liberté, d'anonymat. Un jeune gai de quatorze ans n'a pas de place dans une ville comme Valleyfield. Il ne peut rien y vivre. Il lui faut venir à Montréal. » (Cyr, L'éternité serait-elle un long rêve cochon?, 1997, p. 61). 
la vie nocturne urbaine, ses bruits et la diversité des sous-cultures sexuelles et autres qui s'y retrouvent (prostitution, travestisme, cuir, trafiquants de drogues, punks, etc. ${ }^{16}$ ).

La topographie des espaces de sociabilité et de sexualité où les personnages évoluent recoupe celle rapportée dans les études en géographie urbaine (voir Deligne, Gabiam, Van Criekingen et Decroly 2006, pour la ville de Bruxelles). On y remarque ainsi l'espace du café, qui remplit des fonctions diverses : faire connaissance, retrouver des compagnons de jeux de société, rencontrer des partenaires sexuels ou même avoir des activités sexuelles dans le sous-sol ${ }^{17} \mathrm{Ce}$ sont aussi les bars gais, lieux de sociabilité et de drague associés aux styles vestimentaires (surtout le style cuir) qu'évoquent les auteurs, en y faisant seulement référence ou en décrivant leur atmosphère et leur rôle dans la structuration des sous-cultures gaies, ce que les premiers travaux de sociologie sur le monde gai avaient déjà souligné (Achilles 1967 ; Harry 1974). On retrouve New York avec le Candie, un bar cuir ${ }^{18}$, Montréal avec ses tavernes et ses bars comme le Limelight $^{19}$, Paris avec le Trap, un bar parisien auquel Py (2002) consacre plusieurs pages pour décrire son atmosphère musicale « lourde», l'odeur de transpiration, les couleurs et les jeux de lumière, avec comme métaphore centrale celle de la prédation et de la chasse qui survient dans ce lieu gardé par des portiers qui « résumaient les codes secrets $d u$ lieu. D'une part une fraternité inouïe, de l'autre le danger de l'exclusion [...] $»^{20}$.

Les boîtes de nuit et les raves font aussi l'objet d'évocations de même que les saunas, comme ceux de Los Angeles ou de Montréal sur la rue Saint-Laurent, mentionnés également comme lieux de rencontres ${ }^{21}$ avec leur agencement spatial particulier : des cubicules aux cloisons percées de trous qui permettent des rencontres sexuelles rapides et anonymes ${ }^{22}$, ou des pièces disponibles pour des échanges prolongés. Les endroits de drague ou de rencontres sexuelles plus anonymes comme les dark-rooms, les back-rooms, les toilettes publiques (terminus de bus, universités, haltes routières), que fréquentent des jeunes provenant de petites villes et obligés de cacher leur orientation sexuelle, sont

16. «Les rues de Montréal, l'agitation des rues de Montréal le samedi, passé minuit, les prostituées, les travestis, les vendeurs de dope, les gars de cuir, la jeunesse dorée des banlieues, toute agitée, les clochards, les punks, les cris, les klaxons. » (Cyr, L'éternité serait-elle un long rêve cochon ?, 1997, p. 62).

17. «Je vais souvent dans un café de l'avenue Laurier dont les habitués sont des hommes gais de trente, quarante ans [...] Nous baisons au sous-sol, entre un tas de chaises abîmées et une étagère remplie de conserves, pendant qu'en haut, son chum et associé tient le comptoir. [...] J'y viens pour jouer. Au backgammon, au cribble, au Monopoly. Je recrute des partenaires. C'est une façon de faire connaissance. » (Cyr, L'éternité serait-elle un long rêve cochon?, 1997, p. 91).

18. « De retour à New York j’allai au Candie, un bar cuir sur Amsterdam Avenue. » (White, La symphonie des adieux, 1998, p. 242-243).

19. «Le barman qui me sert un verre avec un point d'interrogation dans les yeux, toujours la crainte d'une descente, d'un contrôle de police. » (Cyr, L'éternité serait-elle un long rêve cochon ?, 1997, p. 62).

20. Py, Paradis de tristesse, 2002, p. 29-30.

21. «André raconte que les clubs de sexe de Los Angeles sont de gigantesques saunas où tout le monde baise avec tout le monde sans préservatifs. » (Cyr, Journal intime d'Éric, séropositif, 2001, p. 93-94).

22. « Je marche jusqu'au boulevard Saint-Laurent. Je m'arrête au sauna. Je m'installe dans un de ces réduits minuscules dont les cloisons sont percées à la hauteur de la taille. Glory Hole. [...] Cloison. Distance. » (Cyr, L'éternité serait-elle un long rêve cochon?, 1997, p. 239). 
aussi indiqués ${ }^{23}$. D'autres toilettes, comme celles du métro ${ }^{24}$, d'une boutique ou d'un avion peuvent servir aux activités sexuelles ${ }^{25}$, tout comme les parcs (Parc Lafontaine à Montréal ; Bois de Vincennes, Parc des Tuileries en France) qui constituent des espaces de drague propices aux contacts sexuels furtifs - favorisés par une végétation abondante et une absence d'éclairage - ou aux conduites exhibitionnistes ${ }^{26}$. Moins fréquemment rapportés, le métro, les ruelles ou les gymnases peuvent aussi favoriser les manœuvres de drague. Les espaces privés (appartements et chambres) sont aussi évoqués, sans trop de détails, dans la construction des scènes intimes.

Le repérage des espaces dominants dans les romans met ainsi en évidence la centralité des grandes villes où se concentrent les populations homosexuelles aux styles de vie diversifiés. Les quartiers, rues, cafés ou saunas occupent une place significative comme espaces de sociabilité dans lesquels circulent les narrateurs ou les personnages des romans qui les fréquentent. Les lecteurs peuvent donc se construire des représentations des lieux imaginaires ou réels proposés dans les textes, évoquer leur ambiance et se sensibiliser à la diversité des styles de vie urbains, surtout homosexuels, ce qui peut les amener par le biais de la fiction à saisir un pan d'une réalité qu'ils peuvent continuer à imaginer ou qu'ils peuvent, dans certains cas, confronter au réel.

\section{L'imaginaire des relations}

Grâce à la densité de sa population, son hétérogénéité et ses constants mouvements, la Citysex (Bech 1999) permettrait d'expérimenter un registre de relations variées qui se fondent sur des principes d'autonomie, de flexibilité et de fluidité. Les romans mettent en évidence la présence de deux grands modèles : celui de la multiplicité des partenaires, essentiellement à des fins sexuelles, et celui de la relation de couple, le plus souvent problématique, où les enjeux sentimentaux et les aspirations à une stabilité, qui n'est pas toujours atteinte, sont prégnants.

Le premier modèle recoupe en partie l'orientation sexuelle définie par Bozon (2001) comme celui du réseau sexuel ou de sociabilité sexuelle où « l'activité sexuelle apparaît aux individus comme une composante ordinaire de leur sociabilité » (2001:16). Si elle peut être « un élément central d'identité sociale », elle n'est pas toujours " génératrice de capital social », « créatrice de liens d'interdépendance » ou constituante d'un « trait d'identité professionnelle » (Bozon 2001 : 16). Dans ce modèle, les individus multiplient les relations et les fondent le plus souvent sur l'anonymat, l'aléatoire et la liberté : ces principes dominent, arrimés au primat de la pulsion et de l'excitation sexuelles, l'exacerbation des sens, l'excès et la recherche de la nouveauté, des éléments qui ne sont

23. «Je parle beaucoup. Du peu de place qu'ont les jeunes gais. Où vivre sa sexualité quand on a quatorze, quinze ans, qu'on habite un village où tout se sait, où tout se tait? Les toilettes du terminus de Montréal, c'est la seule voie, la seule issue ? » (Cyr, L'éternité serait-elle un long rêve cochon ?, 1997, p. 92-93).

24. «Avant de connaître Sean [...], je fréquentais les toilettes du métro pour sucer des types qui rentraient du boulot. » (White, La symphonie des adieux, 1998, p. 48).

25. «Dans l'avion vers Honolulu... je me suis claustré avec lui [un stewart] dans les vécés. » (Cyr, L'éternité serait-elle un long rêve cochon?, 1997, p. 92-93).

26. «Les parcs de Montréal, les bosquets sombres, touffus, les garçons qui circulent à vélo dans les allées sans éclairage, les garçons qui, assis au pied d'un arbre, exhibent leur sexe. » (Cyr, L'éternité serait-elle un long rêve cochon?, 1997, p. 62). 
pas mis en relief par Bozon. Cyr (1997), dans l'un de ses romans, résume les fondements de cette option, en confrontant deux formes d'érotisme dont le premier semble se rapprocher de l'érotisme des corps, « une forme d'égoïsme cynique », selon la définition de Bataille (1957 : 26). Le roulement rapide des partenaires s'inscrit ainsi dans la quête d'une satisfaction pulsionnelle, de l'énervement des sens et dans le maintien d'une tension que ne peut assouvir une relation monogame qui condamne à l'ennui :

Parce que tu le connais son corps à lui, parce qu'il n'y a plus de surprise, plus de mystère, plus de nouveauté. [...] En fait, il y a deux sexualités : celle des sens et celle des sentiments. L'abandon n'est pas le même. L'ivresse, non plus. [...] Il y a deux sexualités. Celle du connu et celle de l'inconnu. [...] Le sexe noir, anonyme, libre et $c r u$, sans romantisme, sans extrapolation, sans attente. Le sexe du présent, animal. ${ }^{27}$

Cette collection insatiable de partenaires, qui s'apparente à une forme de consumérisme noté par Bech (1999 : 219) se retrouve dans plusieurs romans (Gendron 1997 ; White 1998) qui insistent sur le fait que la relation de couple, se fondant sur la domestication de la sexualité, constitue un obstacle majeur à la liberté et au plaisir. Cette relation exige en effet des accommodements quotidiens, jugés dégradants et, de ce fait, elle doit être rejetée car incompatible avec l'expression d'une créativité qui se voit atrophiée par les multiples accrocs inévitables de la vie commune :

[...] l'amour domestique - avec ses mélodrames adultères, ses compromis douillets, ses câlins asexués, ses prises de bec mesquines - me déplaisait précisément parce qu'il puait le possible, le faisable, ce que tout le monde faisait. ${ }^{28}$

Le second modèle se rapproche de l'orientation conjugale (Bozon 2001) sans s'inscrire toujours dans une relation hétérosexuelle ou le mariage. Si, dans des romans, des couples homosexuels ou hétérosexuels semblent réussir à établir une relation stable s'étendant sur plusieurs années ${ }^{29}$, d'autres présentent des narrateurs ou des personnages qui font surtout état des difficultés rencontrées dans leur maintien à cause des enjeux affectifs liés au sentiment amoureux et à la jalousie, aux difficultés de maintenir l'exclusivité relationnelle, ce qui s'accompagne de tensions, d'ambivalences, de rejets et de ruptures, mais aussi d'adaptations difficiles et de compromis qui demandent l'acceptation de relations avec plusieurs partenaires ${ }^{30}$, quelquefois anonymes ${ }^{31}$.

27. Cyr, L'éternité serait-elle un long rêve cochon ?, 1997, p. 121.

28. White, La symphonie des adieux, 1998, p. 442.

29. «Quelques mois plus tard, nous déménageons dans un immense appartement de la rue des Érables. J’y ai mon atelier ; Bruno, son bureau. Nous y resterons sept ans. » (Cyr, L'éternité serait-elle un long rêve cochon?, 1997, p. 103).

30. « J'étais prête à tous les compromis, je me disais : aimer c'est rendre l'autre heureux, alors accepte. Et j'ai accepté tes aventures[...] Tu prends même un malin plaisir à m'imposer tes conquêtes masculines. » (Canesi et Rahmani, Le syndrome de Lazare, 2006, p. 51-52).

31. «Depuis que je vis avec lui, je me permets un plus grand abandon à la sexualité fortuite, aléatoire, celle des parcs, des ruelles et des saunas. [...] Parce que cette présence me rattache à la réalité aussi solidement qu'une ancre. Il est le filet sous le trapèze de mes nuits blanches. » (Cyr, L'éternité serait-elle un long rêve cochon?, 1997, p. 148). 
Les textes relèvent aussi les aspirations à des relations fondées sur la stabilité et l'amour lorsque la maladie frappe et qu'elle remet en question la signification de l'existence :

C'est peut-être la maladie, c'est peut-être tout simplement mon côté romantique, mais j'ai besoin de savoir que l'amour reste possible, qu'il peut durer, transformer la vie, faire grandir. Autrement, rien n'a de sens. ${ }^{32}$

Les romans, sans être très prolixes, illustrent certaines des tensions et des ambivalences qui se retrouvent dans la gestion des relations modernes (Wouters 1999) où le régime monogamique, avec ses valeurs sentimentales et d'exclusivité, s'oppose aux modalités du multipartenariat où domine la quête sexuelle dénuée d'affectivité, donnant lieu à des formes hybrides et métisses qui tentent de réconcilier ces aspirations contradictoires. Comme le souligne Bozon (2001 : 26) :

Au plan intra-subjectif, on observe des clivages internes, qui font coexister ou s'affronter plusieurs interprétations de la sexualité chez un même individu, et des glissements biographiques, qui font se succéder plusieurs perspectives au fil du temps.

Les lecteurs de ces romans peuvent ainsi saisir les enjeux modernes de la relation de couple, de la sexualité ainsi que leurs dimensions affectives et temporelles, enjeux auxquels ils pourraient être confrontés.

\section{L'imaginaire du corps, des affects et des pratiques sexuelles}

Les scénarios entourant le rapport au corps et les pratiques sexuelles occupent une place importante dans ces narrativités romanesques, et l'imaginaire déployé renvoie à un vaste registre affectif et comportemental. Pour décrire les zones génitales et les humeurs corporelles, deux types de lexique sont présents. Le premier renvoie à un langage anatomique (anus, pénis, sexe, testicule, sperme, vulve) et le second à un vocabulaire cru (pine, queue, bite, con, couilles, foutre, par exemple). Cette opposition se retrouve aussi dans la description des pratiques sexuelles avec le recours à un lexique technique (pénétrer, se masturber, sodomiser, être en érection) ou à un langage argotique (baiser, enculer, se branler, bander, faire l'amour, fourrer, faire une pipe, sucer, pomper, juter). Si des descriptions de l'activité sexuelle peuvent être limitées à des notations rapides et peu nuancées, d'autres, au contraire, renvoient à des scénarios plus élaborés, les récits explorant, de façon quasi ethnographique, les zones érogènes, les affects, les pratiques et les gestuelles dont les travaux sociologiques comme ceux de Bolton (1992), Weitman (1999) et Bech (1999) ont montré la diversité.

Le corps

Le rapport au corps se décline de plusieurs façons. Il porte en premier lieu sur l'apparence et sur l'exploration du corps. Bech (1999 : 220) a ainsi noté l'importance du visuel et l'attention orientée sur « les surfaces, les parties et les morceaux [du corps], et non pas sur l'autre personne comme un être humain complet ». La diversité des corps, avec leur potentiel de séduction, se fonde sur la jeunesse, la musculature et l'apparence physique, ces

32. Cyr, Journal intime d'Éric, séropositif, 2001, p. 152-153. 
facteurs étant renforcés par le vêtement ${ }^{33}$. Les codes d'appartenance à des sous-cultures gaies peuvent se définir par des modes vestimentaires, en particulier par l'usage du cuir, qui rehaussent les éléments de l'image corporelle ${ }^{34}$. Les styles vestimentaires peuvent aussi être plus éclectiques, mettant en évidence la diversité des prêts à porter contemporains (jeans, baskets, survêtements, t-shirts, chaps, blouson, etc.) et leur appropriation personnalisée pour attirer l'attention sur des zones corporelles comme les fesses ${ }^{35}$.

Si les diverses parties du corps, dans leur grande majorité, sont mentionnées comme éléments de séduction ou d'aversion, ce sont celles touchant les parties génitales et anales qui retiennent l'attention, avec un accent mis surtout sur la description du pénis, la diversité de ses formes, de sa taille, de son apparence et de la réponse sexuelle liée à l'éjaculation. L'érection et ses difficultés peuvent aussi être soulignées. Les substances corporelles comme la sueur ou le sang menstruel sont peu mentionnés alors que la salive (impliquée dans la lubrification, les baisers et la fellation), mais surtout le sperme (quantité, couleur, texture, etc.), sont plus centraux dans des textes qui valorisent la portée érotique de ce liquide qui peut être avalé, étalé sur le corps ou léché. Les romans tendent donc à insister sur des représentations corporelles essentiellement fondées sur le primat du phallus et du sperme dont on retrouve l'importance dans d'autres textes littéraires pour célébrer l'érotisme homosexuel (Gilles 2009).

\section{Les modalités affectives}

Les activités sexuelles prennent place dans des contextes variés, qui peuvent inclure des échanges de service sexuels ou de la prostitution, et font appel à des modalités affectives diverses : brutalité et soumission d'une part, tendresse et partage, d'autre part, des affects dénotant les oppositions entre une sexualité, qui est axée sur la pulsion exigeant un assouvissement rapide ou, au contraire, qui est sous-tendue par le désir et sa prolongation. On retrouve aussi des tonalités de fébrilité et de fièvre, d'espoir et de désespoir, des tentatives de jonction entre sexualité et amour et, à l'inverse, leur déliaison. Les affects de honte et de culpabilité liés aux activités anonymes font l'objet de jugements contradictoires. Ils peuvent ainsi dominer et affecter l'estime de soi ou ne jouer aucun rôle dans la gestion de la sexualité ${ }^{36}$. La quête de l'avilissement comme mode d'exploration intérieure et de

33. «On sortait au BH, la boîte Crash de la rue du Roule. Vingt-quatre et vingt-sept ans, pas un gramme de graisse, les cheveux très courts, parfaitement lookés. On était les plus beaux. On ramenait des mecs. » (Dustan, Plus fort que moi, 1998, p. 45).

34. «Vêtements conçus comme de la publicité érotique [...] leurs corps sans parfum moulés, en plus, par le cuir noir dans la mesure où seul le look sadique allait bien avec l'extrême pâleur. » (White, La symphonie des adieux, 1998, p. 254-255).

35. "L'un porte des jeans découpés en L sur la raie des fesses et sous la fesse droite. » (Dustan, Plus fort que moi, 1998, p. 91-92).

36. «Il lui arrive, à lui aussi, de fréquenter les parcs et les toilettes publiques. Mais il en ressent toujours une certaine honte, une culpabilité. Ces « écarts » entachent l'estime qu'il voudrait avoir de lui-même. Il s'y livre à son corps défendant. Ou plutôt à son esprit défendant, sa tête admettant difficilement ce que son corps réclame. Moi, je n'éprouve ni honte ni culpabilité. Nous avons des façons différentes, opposées, de gérer les mêmes pulsions. " (Cyr, L'éternité serait-elle un long rêve cochon ?, 1997, p. 119). 
connaissance de $\operatorname{soi}^{37}$, mais aussi paradoxalement comme obtention d'une forme de pouvoir au prix de sa dignité, peuvent étayer les activités sexuelles anonymes.

Et il faut avoir pratiqué cette forme de sexualité débridée, ouverte, gourmande, constante, pour savoir à quel point elle donne du pouvoir. Un pouvoir immense, démesuré. [...] En me livrant comme ça à de parfaits étrangers, j'acquérais un pouvoir phénoménal. En me pliant à tous leurs caprices, je les dominais. En devenant leur chose, je les contrôlais. En me soumettant à eux, je les tenais à ma merci. [...] Le pouvoir a un prix. Le pouvoir du cul s'obtient au prix de la dignité. ${ }^{38}$

Quant à la compulsion sexuelle, peu traitée, elle est considérée comme une stratégie pour trouver une source de valorisation et échapper ainsi à l'emprise d'affects négatifs :

Dans cette compulsion, il y avait du chagrin, évidemment, du dépit, de l'écœurement, du désespoir aussi, beaucoup de désespoir. Et le cul, ça me permettait de me valoriser. Dans le sexe, on cherche toujours à se valoriser. Ça nous dit qu'on est beau, désirable. Le sexe, ça flatte l'ego. ${ }^{39}$

Dans ces romans, l'intrication des affects et des motivations qui accompagnent l'exercice de la sexualité met en relief une réflexivité qui peut amener les lecteurs à explorer les différentes significations de l'érotisme, mais aussi ses dimensions sensibles et métisses qui renvoient à une tension constante qui ne peut être résolue (Laplantine 1997). Cette dimension sensible se prolonge par l'exploration des modalités sensorielles impliquées dans la sexualité et qui n'ont pas fait l'objet d'études approfondies malgré l'importance des sens dans la construction des individus et dans les cultures (Le Breton 2006).

\section{Les modalités sensorielles}

Les modalités sensorielles dont il est question concernent le regard et la voix, l'olfaction et le goût de même que le toucher.

\section{Le regard et la voix}

La modalité visuelle est évoquée dans les rituels de drague ou de rencontre et fait référence au ballet des regards et des codes gestuels de reconnaissance et d'invitation, ainsi qu'aux affects fluctuants de l'attirance et de la peur qui accompagnent les échanges qui peuvent survenir dans des endroits publics comme une rame de métro ${ }^{40}$ ou la rue :

37. « Je m'exorcise, je joue, je m'avilis s'il me vient l'envie de m'exorciser, de jouer, de m'avilir. Je veux voir jusqu'où je peux aller là-dedans. J'ai besoin de savoir qui je suis, de savoir quelle est cette part de moi, de l'explorer, de la reconnaître comme j'explore et reconnais les images et l'âme des autres. Pourquoi broder? Je passe au sexe de plain-pied, si je puis dire. » (Cyr, L'éternité serait-elle un long rêve cochon?, 1997, p. 122).

38. Cyr, Journal intime d'Éric, séropositif, 2001, p. 157.

39. Cyr, Journal intime d'Éric, séropositif, 2001, p. 157.

40. « Vous prenez le métro [...] Puis, vous voyez ce garçon assis en face de vous, [...] qui lève constamment les yeux de son bouquin pour regarder bien droit entre vos deux jambes. Il tire un peu sur la fourche de son jeans pour révéler qu'il est bandé. [...] Il descend à la station suivante. Vous le suivez. » (Cyr, L'éternité serait-elle un long rêve cochon?, 1997, p. 122). 
Il y avait la drague dans la rue avec son rythme de plus en plus rapide d'échange de regards, le contact visuel apparemment accidentel cédant la place à un examen minutieux et prolongé, la chorégraphie invariable unissant les danseurs en une valse hésitation de désir et de peur. ${ }^{41}$

L'importance du regard se manifeste aussi dans les interactions érotiques entre les protagonistes qui transmettent des affects variés (amour, tristesse, douceur, sollicitude, colère, etc.) ou qui établissent un rapport de domination-soumission : "Ce regard me demande si je donnerais tout. Ce regard vérifie que je donnerai tout. Ce regard se regarde lui-même dans le miroir de ma soumission. ${ }^{42}$

La charge érotique de la voix est peu rapportée, mais les romans font mention des modulations vocales qui accompagnent l'activité érotique (grognements, geignements, cris, râles) qui peuvent servir de source d'excitation : "Hubert entre en moi en râlant [...] puis me mord. Je crie : en général, ça l'excite... Il jouit. $»^{43}$

\section{L'olfaction et le goût}

L'olfaction et la qualification des odeurs des parties du corps ou des vêtements jouent aussi un rôle important dans l'induction de l'excitation sexuelle et renvoie aux senteurs des zones corporelles ou des vêtements, dans une palette variée : "L'odeur du sel dans ses cheveux et sur ses épaules. L'odeur de ses aisselles. [...] L'odeur de son sexe. " ${ }^{44}$; "J'ai le visage au niveau de sa ceinture, une odeur de poivre et de menthe m'envahit, l'odeur de ses vêtements. ${ }^{45}$. Les odeurs des substances corporelles fondent aussi la puissance reconnue à un slip d'homme associé à des pratiques fétichistes : "Mais avant tout ce fétiche est odorant [...] je l'embrasse, n'y laisse pas trop longtemps la bouche de peur de corrompre son odeur, je jouis. [...] Idolâtre fou de cette odeur de pisse et de sueur et de sexe que son slip recueille $[. ..] »^{46}$. Le goût est aussi impliqué dans les rencontres érotiques, et les saveurs spécifiques, excitantes ou aversives, des zones érogènes sont évoquées : "Il se souvenait du goût brûlant et amer de concombre desséché de son anus. $»^{47}$; "J'ai dans la bouche son goût de fer et de lait. $»^{48}$; «Après on s'est sucé. Le goût était horrible. $»^{49}$

41. White, La symphonie des adieux, 1998, p. 26.

42. Py, Paradis de tristesse, 2002, p. 85-87.

43. Canesi et Rahmani, Le syndrome de Lazare, 2006, p. 15.

44. Cyr, L'éternité serait-elle un long rêve cochon ?, 1997, p. 46.

45. Py, Paradis de tristesse, 2002, p. 40.

46. Py, Paradis de tristesse, 2002, p. 143-144.

47. White, L'homme marié, 2000, p. 36.

48. Py, Paradis de tristesse, 2002, p. 85.

49. Dustan, Plus fort que moi, 1998, p. 15. 


\section{Le toucher}

La dimension tactile n'est pas non plus négligée et, si elle traverse l'ensemble des scènes érotiques, elle se retrouve en particulier dans les activités que Weitman (1999 : 84) définit comme les self-entrustements pour désigner les différentes modalités de touchers comme les contacts des mains et les autres gestes manuels qui accompagnent les interactions érotiques (caresses, frottements, palpations, pressions, pétrissage, pincements, morsures, etc.) et qui portent sur l'ensemble du corps ou des parties précises, en particulier les zones génitales. Les caresses buccales, le léchage des parties du corps, du derrière, de l'anus (rimming) ou des testicules, le têtage ou les contacts corporels peuvent servir de préliminaires ${ }^{50,51,52}$. Cet univers sensoriel accompagne l'expression d'un large registre de pratiques sexuelles qui rejoignent la nomenclature dégagée par Bolton (1992), à partir d'une enquête auprès d'informateurs gais et qui comprend une liste de près de 100 énoncés renvoyant aux conduites érotiques. Bolton propose de les appeler des « sexèmes » ou des « sexons », « c'està-dire les unités élémentaires de comportement qui constituent des éléments significatifs (meaningful) dans les rencontres sexuelles » (Bolton : 145).

\section{Les sexons}

Pratiquement tout l'ensemble des « sexons », définis par Bolton (1992), se retrouve dans les narrations qui en explorent les différentes facettes.

\section{Le baiser}

On peut ainsi repérer les références aux baisers sur les lèvres (« [...], je remplis sa bouche de ma langue $»^{53}$ ou plus profonds, renvoyant à des métaphores de type énergétique («Un baiser est une fusion nucléaire $"{ }^{54}$, ou encore aux échanges de salive qui constituent le signe d'une communion : «les [...] partenaires hétérosexuels communient par leur salive échangée. $\rrbracket^{55}$. Le baiser prolongé devient aussi l'expression d'un lien amoureux entre deux partenaires gais, ${ }^{56,57}$ mais il se retrouve aussi dans un contexte anonyme lié à des pratiques

50. « Je suis tout près de lui, je caresse son dos humide et chaud. [...] je glisse ma main sous son caleçon, palpe ses fesses. » (Canesi et Rahmani, Le syndrome de Lazare, 2006, p. 82).

51. «M'a présenté son cul. J'ai mis le nez au creux de sa raie. [...] J'ai léché à lents coups de langue, de l'entrecuisse à juste au-dessus du trou [...]» (Dustan, Plus fort que moi, 1998, p. 110).

52. «Il [...] se love derrière moi, effleure le bout de mes seins, glisse ses mains sous mon chemisier, me caresse les hanches, le ventre, la poitrine. Je tremble de plus belle [...] » (Canesi et Rahmani, Le syndrome de Lazare, 2006, p. 250-251).

53. Canesi et Rahmani, Le syndrome de Lazare, 2006, p. 82.

54. White, La symphonie des adieux, 1998, p. 280.

55. Deroche, Effets secondaires, 2002, p. 68-70.

56. «Austin n'avait connu pareille étreinte - chaque baiser empreint de la volonté d'absorber l'autre. » (White, L'homme marié, 2000 : 127).

57. « La nuit la plus romantique de ma vie je l'ai passée avec un homme plus âgé dans les dunes de Fire Island, à l'embrasser jusqu'à ce que mon visage soit tout irrité par sa barbe [...]» (White, La symphonie des adieux, 1998, p. 349). 
de type orgiaque où il peut être refusé, à cause de l'association aux infections comme l'herpès ou, au contraire, acceptés ${ }^{5}$. Ces notations recoupent les travaux sur l'histoire et les significations du baiser qui renvoient à des connotations romantiques, communielles, mais aussi hygiéniques (Médico-Vergriete et Lévy 1999).

\section{Les pratiques bucco-génitales}

Les pratiques bucco-génitales, surtout homosexuelles, peuvent être décrites de façon stéréotypée (sucer, pomper, prendre dans la bouche) ou être plus détaillées quant aux techniques et aux gestes concomitants, absorption du sperme ou refus de l'ingestion, masturbation et éjaculation, par exemple ${ }^{59}$. Ces pratiques peuvent survenir dans des rapports personnalisés ou plus anonymes dans le contexte des saunas ${ }^{60}$, du métro, des toilettes publiques ou des parcs, et s'accompagner, dans certains cas, de formes de coercition ou de brutalité ${ }^{61}$. On retrouve aussi dans l'un des romans, un éloge du cunnilingus lors de la période de menstruations pour le plaisir qu'il procure, le sang menstruel constituant une substance appréciée qui peut être ingérée ou utilisée comme peinture corporelle ${ }^{62}$.

\section{Les pratiques masturbatoires}

La fellation, comme nous l'avons vu, peut être concomitante à des pratiques masturbatoires, mais ce sont surtout les activités allomasturbatoires ou mutuelles qui sont rapportées dans des contextes de rencontres anonymes dans les parcs ou lors de relations plus personnalisées. Les gestes (contacts des pénis, massages, frottements) ${ }^{63}$ et les réactions

58. «Et puis tout d'un coup une bouche s'est collée à la mienne. Je n'avais aucune idée de celui à qui elle pouvait appartenir (un vieux !, l'herpès !). J'ai détourné la tête d'un coup sec. [...] Je me faisais palper de tous les côtés et puis le mec qui était face à moi a cherché à m'embrasser et comme il était correct je me suis laissé faire. » (Dustan, Plus fort que moi, 1998, p. 20-22).

59. «Sa bouche, sa salive, tout autour de mon pénis, qui va et qui vient dans sa bouche, déjà mon dieu, un éclair bleu, il avale tout en se masturbant jusqu'à ce que filent de sa queue de longues giclées argentées qui retombent sur le carrelage blanc, constellé de sable. » (Cyr, L'éternité serait-elle un long rêve cochon, 1997, p. 46).

60. «Je m'arrête au sauna. [...] Une queue jaillit de la cloison. Et je la prends dans ma bouche. » (Cyr, L'éternité serait-elle un long rêve cochon, 1997, p. 239).

61. «Bel uniforme ! [...] il se sort la bite et sa main sur mon épaule m’indique le reste. Je m'agenouille et je fais ce qu'il demande. [...] Tant qu'il est dans ma bouche, sa matraque, son insigne ne tiennent plus, ne veulent plus rien dire. » (Cyr, L'éternité serait-elle un long rêve cochon, 1997, p. 72).

62. «Le plaisir est double : on tète le clitoris, on lape les replis cutanés de la vulve, voire le pourtour de l'anus. Ainsi s'assouvit cette faim de connaissance du corps étranger. [...] le sang des menstrues ne fait pas reculer le brave. [...] on absorbe les sécrétions avec avidité. Le connaisseur s'en oint les lèvres, les joues, le nez, avec la même gourmandise que l'enfant qui se barbouille avec le nappage de son sundae fraise. » (Deroche, Effets secondaires, 2002, p. 68-70).

63. «Une nuit. Parc La Fontaine. Un homme dans les buissons. [...] Qui masse doucement mon sexe en regardant tout autour de nous pour s'assurer que personne ne vient. » (Cyr, L'éternité serait-elle un long rêve cochon, 1997, p. 69). 
de plaisir qui accompagnent ces pratiques sont ainsi esquissées, culminant avec l'éjaculation sur le visage ${ }^{64}$ ou dans les mains, suivi de l'emploi du sperme comme enduit corporel ${ }^{65}$.

\section{Les pratiques digito-anales et le fisting}

Les pratiques digito-anales avec l'insertion des doigts ${ }^{66}$ et du poing dans l'anus (fisting), ou d'autres objets ${ }^{67}$, sont aussi rapportées, quelquefois avec le détail des gestuelles impliquées (poussées, mouvements et positions des mains) et la mention des substances (poppers et lubrifiants) pour faciliter la pénétration ainsi que les réactions du partenaire ${ }^{68,69,70}$. Les risques de blessures et de saignements sont mentionnés, sans constituer cependant une préoccupation importante.

\section{Les pratiques interfémorales et anales}

Les romans font mention de relations interfémorales (insertion du pénis entre les cuisses du partenaire), mais ce sont surtout les relations anales qui sont décrites en faisant référence aux dimensions comportementales et, dans certains cas, aux affects comme la surprise liée à l'absence de douleur et l'étonnement face au plaisir ressenti ${ }^{71}$. Si, dans la plupart des scènes érotiques, la finalité du plaisir est recherchée, sa narration est le plus souvent limitée à l'usage de mots convenus, comme « jouir » ou « orgasme », le lexique le plus fréquent, ou peut renvoyer à d'autres expressions plus rares comme « venir ».

64. «Le sofa est bien étroit. Il ne fait pas d'histoires. Je lui éjacule en plein visage. C'est pour lui la première fois. Quoi, il croyait que ça n'arrivait que dans les revues de cul ? Il ne fait pas d'histoires. » (Cyr, L'éternité serait-elle un long rêve cochon, 1997, p. 103).

65. «Louis joignit son sexe au mien dans ses mains et les massa ensemble en leur imprimant un mouvement de va-et-vient de plus en plus rapide. [...]Nous jouîmes ensemble dans ses mains.— Ah ! ton foutre ! Que c'est bon ! J'aime ! J'aime ! Et il se frottait les lèvres, la gorge, la poitrine, le ventre de ses mains gluantes. Il se pourléchait les doigts. » (Henri, Bleu Caraïbes, 2004, p. 52).

66. «Par petits mouvements circulaires, j'entre en lui mon majeur enduit de salive, il se contracte autour de mon doigt. » (Canesi et Rahmani, Le syndrome de Lazare, 2006, p. 82).

67. « Il m'a refourré l'œuf bien au fond avec deux doigts, et puis il a rerentré sa main pour aller le chercher une deuxième fois. [...] Le ramassage des œufs, encore un truc que je n'avais jamais fait. » (Dustan, Plus fort que moi, 1998, p. 55).

68. «Il m'avait même enculé avec son poing un après-midi à Fire Island alors que nous étions tous deux défoncés et immergés dans une mer de graisse. » (White, La symphonie des adieux, 1998, p. 97).

69. «Empalé sur mon poing tu te défonces en geignant telle une statue en transe [...]» (Gendron, Le prince des Ouaouarons, 1997, p. 17).

70. « [...] il a ouvert le sac, dévissé le poppers, sniffé sans s'arrêter pendant que je poussais [...] sans avoir à détendre les parois en faisant les ciseaux avec les doigts, pas une seule contraction des sphincters, jusqu'à ce que ma main soit logée à l'intérieur et que son cul se referme sur mon poignet. Dedans j'ai ramené mes doigts sur ma paume. J'ai fermé le poing. J'ai commencé à tourner sur la droite, sur la gauche. » (Dustan, Plus fort que moi, 1998, p. 94).

71. « Il se plaque violemment sur moi, [...] et au début je suis surpris de la brutalité qui est la sienne quand il entre en moi [..] mais surtout de l'absence de douleur, étonné par ce plaisir qui rapidement monte, [...] qui vient du plaisir que lui-même prend à me garder immobilisé sous son poids et qui vient de la dure poussée de ses reins. » (Canesi et Rahmani, Le syndrome de Lazare, 2006, p. 82). 
La conclusion des séquences érotiques obéit à des modèles variés. Dans le cas des relations anonymes et furtives, l'activité sexuelle se termine avec l'acmé sexuel, suivi de la séparation précipitée des partenaires. Dans certains scénarios, cette phase donne lieu à des modalités de distanciation rapide de la part des partenaires ou, au contraire, à des formes d'expression émotive jugée déplacée et désagréable ${ }^{72}$. Par contre, dans le cadre de relations personnalisées, les scénarios sont plus variés. Ainsi, les partenaires peuvent prolonger le contact physique, en écoutant la musique ou s'endormant ensemble, dans une étreinte ${ }^{73},{ }^{74},{ }^{75}$. Cette phase peut être aussi le moment de faire plus ample connaissance et s'accompagner d'échanges sur les itinéraires personnels, la vie familiale, amoureuse et sexuelle ou les projets d'avenir ${ }^{76,77}$ :

Nous parlons longuement après avoir baisé. Nus tous les deux sous un drap mince dans le lit simple, éclairés seulement par une bougie, nous déconnons, rions, fumons ses cigarettes. Il me parle de son père, je lui parle du mien. Il me raconte ses expériences sexuelles, je lui raconte les miennes. [...] Nous parlons de sexualité clandestine, souterraine, anonyme. De sexualité animale, primaire. Nous nous demandons en souriant si nous sommes normaux. ${ }^{78}$

72. «Après la jouissance, certains deviennent durs et froids et distants, s'en vont tout de suite, honteux. D'autres deviennent tout mous, sucrés et finalement écœurants de douceur paternaliste. » (Cyr, L'éternité serait-elle un long rêve cochon, 1997, p. 64).

73. « À la fin, ils restèrent couchés là sans bouger, écoutant la musique. » (Maupin, D’un bord à l'autre, 1997, p. 243).

74. « Ses doigts glissèrent sur mes flancs apaisés. Nous nous endormîmes de concert. » (Henri, Bleu Caraïbes, 2004, p. 15-16).

75. « Sur le sofa trop étroit, je m’endors dans ses bras. » (Cyr, L'éternité serait-elle un long rêve cochon, 1997, p. 103).

76. «Bizarrement, sa curiosité naturelle pour les gens se manifestait après le sexe. [...] il me posa toutes sortes de questions sur moi, non pas avec la concentration avide de celui qui vous tourne autour mais plutôt avec l'affabilité mâcheuse de chewing-gum d'un pote qui a les yeux fixés au plafond. » (White, La symphonie des adieux, 1998, p. 281).

77. «La phrase « sexe anonyme » peut suggérer sexe sans sentiments, privé d’émotion. Et cependant, ainsi que je peux l'attester, se planquer dans une pièce d'un bain public avec un corps [...] la tête posée sur le ventre d'un type[...] et parler jusque tard dans la nuit et tôt le matin, de notre enfance, de ses malheurs en amour, de nos problèmes d'argent, de ses projets d'avenir - eh bien, rien n'est plus personnel, plus émouvant. Le meilleur de tout c'étaient les pensées vagabondes et flottantes que nous partagions. » (White, La symphonie des adieux, 1998, p. 349).

78. Cyr, L'éternité serait-elle un long rêve cochon, 1997, p. 56. 


\section{Les paraphilies}

Les pratiques sadomasochistes sont aussi mentionnées, mettant en évidence, à part le bondage, des conduites de soumission et d'humiliation ${ }^{79,80}$. Le fétichisme, comme nous l'avons déjà mentionné, est aussi présent, comme les pratiques urophiles, le voyeurisme, la pédophilie ou les pratiques de type orgiaque. Ces dernières visent à la quête des états de conscience modifiés recherchés à travers la musique, la danse et le recours aux drogues comme le speed et l'ecstasy, des éléments des rituels orgiaques modernes qui permettent l'atteinte d'une transe, l'oubli de la fatigue et de soi $^{81,82}$. Les participants peuvent aussi s'impliquer dans plusieurs pratiques sexuelles (palpations, baisers, fellations, relations digito-anales, etc.) avec des partenaires multiples anonymes. L'obscurité contribue aussi à amplifier l'excitation rattachée à ces multiples contacts et à l'effervescence érotique, même si des affects de honte peuvent aussi être présents ${ }^{83}$.

\section{Les pratiques médiatisées}

Quant aux espaces télémédiatisés, auxquels Bech (1999) fait allusion dans sa définition de la Citysex, les romans en font quelques mentions : emprunt d'une pratique sexuelle suite au visionnement de films pornographiques américains ${ }^{84}$, recours aux rencontres téléphoniques et à la pornographie pour éviter les risques de transmission du VIH/sida, mais en insistant sur le côté dérisoire de telles stratégies :

Beaucoup trouvaient un soulagement au téléphone, payant des sommes importantes pour atteindre le nirvana. Le sexe par téléphone, avait remarqué Michael, non seulement développait l'imagination mais donnait aussi la possibilité aux hommes

79. «Il m'a montré qu'il était le maître, simplement en exhibant sa queue, en me l'interdisant, qu'il n'ait pas bandé vraiment accentuait la force rituelle de son geste, il me présentait, présenter est le verbe juste, sa puissance, et je m'étais agenouillé devant sa puissance. » (Dustan, Plus fort que moi, 1998, p. 111).

80. «Ellert est nu sur le lit. Pascual lui écarte les jambes, cette inspection lente humilie furieusement Ellert [...] Pascual le gifle et lui ordonne de se taire. [Il] l'attrape par les cheveux et le force à lécher ses chaussures [...] Pendant qu'il lèche, Pascual prend sa ceinture et lui frappe le trou du cul. Le visage d'Ellert est tout près du mien, au sol, je vois ses yeux pleins de larmes, sa bouche qui bave. » (Py, Paradis de tristesse, 2002, p. 95).

81. «Je me souviens comme il dansait, comme un fou, dans la boîte pédé de Valence, sur le tube house que nous préférions : Es imposible, no puede ser. » (Dustan, Je sors ce soir, 1997, p. 13-14).

82. « Je voudrais avec toi retourner au Stéréo ou dans un rave illégal, comme ceux où nous allions danser toute la nuit, sur le speed et l'ecstasy, [...] danser comme deux automates sur le rythme hypnotique de la transe [...] inconscients de l'heure, de la fatigue, de la douleur dans nos jambes, inconscients tout court, indifférents au monde, hébétés, absents et heureux de l'être. » (Delorme, Afin que personne ne puisse nous faire de mal, 2001, p. 63).

83. « Je me faisais palper de tous les côtés [...] Je pouvais m’engloutir dans ce magma de mains, de bites, de bouches. Je pouvais me mettre à ne plus rien en avoir à foutre de savoir à qui appartenait quoi, qui était gros, vieux, moche, contagieux. Je pouvais [...] devenir une bête, ressortir des heures après, les vêtements déchirés, tachés, nu, couvert de sueur, de salive, de sperme. [...) Je me suis redressé d'un bond, les larmes aux yeux. J'ai trébuché jusqu'à la sortie en retenant mon jean avec les mains. Je me suis rhabillé sur le seuil, le cœur battant, sans oser regarder devant moi. » (Dustan, Plus fort que moi, 1998, p. 20-22).

84. « [...] après je lui ai demandé où il avait appris ça [claquer les fesses], il m'a répondu dans les films américains, j’ai rêvé sur la culture mondiale. »(Dustan, Plus fort que moi, 1998, p. 61-62). 


\section{de faire une chose qui jusque-là ne leur était pas possible : faire semblant d'avoir un orgasme. $^{85}$}

Si les pratiques cybersexuelles sont absentes des romans tout comme l'usage de gadgets érotiques, on retrouve des références aux outils associés aux nouvelles technologies de communication comme le Minitel, un réseau informatique français aujourd'hui disparu, l'internet et le téléphone portable qui servent à rencontrer des partenaires ${ }^{86}$ avec des profils spécifiques, comme le barebacking ou le sadomasochisme ${ }^{87,88}$. La masturbation sans partenaire peut aussi survenir, associée à des fantasmes renvoyant aux moments érotiques vécus avec des partenaires rencontrés dans le passé ou à l'usage d'images pornographiques ${ }^{89,90}$.

En conclusion, les romans permettent de cerner certaines des modalités affectives entourant l'expression érotique et de mettre à jour ses fonctions psychologiques et les dilemmes qui lui sont associés. Les scénarios rattachés aux conduites sexuelles, tout en insistant sur les dimensions sensorielles, rejoignent dans leurs grandes lignes ceux dégagés dans la littérature sociologique auprès des populations homosexuelles (Bolton 1992). Dans l'ensemble sans grande originalité, ils pourraient contribuer à renforcer des représentations de la sexualité basée sur la performance et la quête du plaisir et à orienter les lecteurs vers ces valeurs dominantes qui semblent aujourd'hui moins subversives, rejoignant ainsi les normes présentes dans les courants littéraires (Pivert 2009).

\section{L'imaginaire des risques et de la prévention}

La question du VIH/sida et ses répercussions sur l'expression érotique, la prise de risques et les stratégies de prévention constitue l'un des thèmes majeurs de ces romans. Plusieurs pages sont consacrées aux enjeux entourant la gestion complexe du préservatif et le dévoilement du statut d'infection, des préoccupations qui font écho aux travaux sociopsychologiques sur cette question (Paxton 2002). Les textes mettent en évidence les ambivalences entourant les consignes de précaution prônées par les instances de santé publique et présentent des scénarios où la recherche du risque et de la contamination volontaire sont valorisés, subvertissant ainsi les mots d'ordre du sexe sécuritaire critiqué par plusieurs romanciers. Si le thème de la mort continue d'être prégnant, sans atteindre l'intensité relevée dans les romans plus proches du début de l'épidémie (Lévy et Nouss 1994), le risque d'être infecté est ainsi envisagé comme une source d'excitation, une forme

85. Chabot, Innocence, 2007, p. 40-41

86. « Je fais du net, je collectionne les numéros de portable et les adresses [...], ils sont tous sur le haut débit [...]» (Dustan, Génie divin, 2001, p. 28).

87. «C'est génial parce que sur le net nous les gens qui ont quelque chose en commun, le bareback par exemple, on peut tous se retrouver. » (Dustan, Génie divin, 2001, p. 138-139).

88. «Les jours suivants j’ai cherché sur minitel. J'ai fini par tomber sur un mec passif, soumis, la quarantaine, assez moche mais hyper-docile. » (Dustan, Plus fort que moi, 1998, p. 76).

89. « Austin admettait être plus attiré par ce dernier - il pensait à lui toutes les nuits, et se masturbait encore en songeant à lui deux fois par jour. » (White, L’homme marié, 2000, p. 36).

90. «Austin avait acheté des revues porno et les utilisait scrupuleusement pour se masturber, bien que la fixité des photographies lui déplût (il préférait les films cochons ou, mieux encore, les histoires). » (White, L'homme marié, 2000, p. 230). 
de conduite ordalique qui accompagne les conduites extrêmes et qui permet d'atteindre à un surcroît d'existence (Le Breton 1991). La relation entre éros et thanatos est ainsi clairement établie en recourant à la métaphore classique de la roulette russe qui renvoie au jeu de l'aléa (Caillois 1958) privilégiant le recours à la chance et à la victoire sur le destin (Lévy 1996) :

Chose certaine, notre sexualité flirte pas mal avec la mort. Pensez seulement à ceux qui courent après la maladie. [...] Pensez au barebacking [...] très en vogue en Amérique. Nouvelle tendance. Pensez à tous ceux qui, conscients des risques et à cause justement des risques, n'hésitent pas à en prendre. Parce que les risques, ça crée une excitation supplémentaire, on accède à un autre niveau, on se détache du commun des mortels, c'est comme jouer à la roulette russe. Le cul, sport extrême. Pourquoi la mort dans le sexe nous fascine-t-elle tant ? ${ }^{91}$

La question du barebacking fait l'objet de nombreuses réflexions visant à le définir et à dégager les enjeux éthiques associés à ce type de pratique en portant les débats sur le terrain de la liberté et de la responsabilité individuelle, en refusant des formes de contrôle répressif de la sexualitée ${ }^{22}$, y compris de la part des instances associatives orientées vers la prévention comme Act $U p$, et en modulant la prévention en fonction du statut sérologique des partenaires et de la relation.

La prise de risques peut aussi ne pas se situer dans une perspective réflexive et correspondre à une forme d'insouciance, à une perception du VIH/sida comme une maladie aujourd'hui bénigne suite aux innovations liées aux traitements antirétroviraux:

Le seul tuyau pour être bien tranquilles : pas de tests, pas de capotes [...] De toutes façons, c'est tellement con (depuis les traitements, surtout), tellement con d'être séroneg [...] De "faire attention »; tout le temps. [...] Du temps de Baudelaire, on ne faisait pas tant de manières avec le cul, non mais! On la chopait, la syphilis, et puis on la refilait, à qui qu'en voulait... The bug, comme disent les barbaqueers, là-bas... Gimme the bu-u-ug, and 1 will be free-ee-ee! No more worry-y-y. ${ }^{93}$

L'ignorance des risques, l'acceptation de l'infection au virus qui met fin aux préoccupations entourant la prévention ou même l'acceptation fataliste de la mort constituent aussi des modalités d'un rapport moins prudent face au virus ${ }^{94}$. La prise de risque apparait aussi comme une façon d'échapper à la conformité du monde ambiant en privilégiant un style de vie basé sur l'excès, qui serait pour certains l'un des traits de la modernité (Arcand

91. « François n'a jamais voulu se protéger, casse-cou de l'amour, pas de filet, peu de capotes. Il s’en fout. “Ce n'est pas grave", il est sûr de lui. »(Cyr, Journal intime d'Éric, séropositif, 2001, p. 156).

92. «Je ne suis pas un prosélyte du barebacking. Mon combat se situe au niveau de la liberté et de la responsabilité individuelle. Je suis contre toute répression de la sexualité et des libertés individuelles surtout par la culpabilité, la honte, la morale et la terreur. Le rôle de l'écrivain est aussi de mettre en garde, de poser des questions violentes. Face à l'irrationalité du sexe, il s'agit donc de ne pas avoir de positions trop tranchées, mais de faire preuve de souplesse. » (Rémès, Serial fucker : journal d'un barebacker, 2003, p. 173).

93. Dustan, Génie divin, 2001, p. 131-137.

94. « Non, non, dans mon pays, l'Espagne (je suis ibère d'origine), on ne fait pas de tests (c'est pour ça, figurezvous, que la "prévalence de l'épidémie" y est moins forte que chez vous, les Français). Dans mon pays, on n'en met pas, des capotes. [...] Et puis on meurt. Voilà. De ce qui fait mourir. C'est tout. » (Dustan, Génie divin, 2001, p. 131-132). 
1991), que ce soit dans le champ de la sexualité ou de la consommation des drogues et de l'alcool ${ }^{95}$, laquelle contribue souvent aux conduites sexuelles à risques et aux infections transmises sexuellement (Lévy 2006). Ces infections sont illustrées dans les romans avec des mentions de la blennorragie (pharyngée, rectale ou pénienne), dénotée argotiquement (chtouille) et liée à la multiplicité des partenaires sexuels, mais elle ne fait pas l'objet, même lorsqu'elle est répétée, de préoccupations particulières ni d'affects de honte ou de culpabilité, des antibiotiques étant disponibles pour le traitement ou pouvant être pris à titre préventif. Les risques de mutations ou de résistance des bactéries sont ainsi envisagés avec sérénité, les doses d'antibiotiques pouvant être augmentées pour contrer ces conséquences ${ }^{96}$.

Ces infections apparaissent, dans cette perspective, ne plus constituer des évènements graves dont il faut cacher l'occurrence, mais des inconvénients banals facilement traitables avec les médicaments disponibles.

Dans ce contexte l'inclusion du préservatif dans les scénarios sexuels renvoie à des cas de figure variés. Suite à une simple demande du partenaire, il peut être intégré sans discussion aux activités sexuelles ou susciter des demandes pour expliciter les raisons qui en motivent l'usage comme par exemple la multiplicité des partenaires ou la fragilité de la relation. Les difficultés de communication quant au dévoilement du statut d'infection et à la prévention donnent ainsi lieu à plusieurs hypothèses contradictoires qui soulignent la complexité des interprétations proposées pour expliquer le non-usage du préservatif lors de relations anales :

Il a voulu m'enculer sans condom. J'ai patiné, j'ai bafouillé. "On ne se connait pas beaucoup. Et puis, on est instables ». [...] Il ne s'est pas formalisé. Il a déchiré d'un coup de dents une petite enveloppe carrée [...] Mais, moi, je n'étais plus vraiment là. [...] Qu'est-ce que ça veut dire quand un gars se propose de baiser sans condom [...] ? Qu'il est séronégatif et naïf? Ou qu'il est séropositif et dangereux? C'est de la candeur ou bien de la témérité ? Est-ce qu'il devine que je le suis et cherche à me révéler qu'il l'est aussi ? Ou exactement le contraire : qu'il n'a rien et suppose que je n'ai rien non plus ? [...] J'ai échafaudé toutes sortes d'hypothèses, qui se valent les unes comme les autres, et je ne suis arrivé à rien. ${ }^{97}$

D’autres extraits de romans indiquent le rejet du préservatif qui peut être abandonné à cause de ses effets anhédoniques jugés trop lourds sur la vie érotique, même après des

95. «Parce que le risque était pour moi un mode de vie, parce que le risque me semblait plus beau, plus méritoire même, parce qu'il était un déni du confort facile des bien-pensants, des apôtres du conformisme. Je n'ai jamais fait attention à rien. J'ai baisé sans condoms mais aussi j'ai bu et me suis gelé jusqu'à la démesure. » (Delorme, Afin que personne ne puisse nous faire de mal, 2001, p. 80).

96. «J'avais des rapports sexuels avec tant d'hommes, souvent un nouveau chaque soir, qu'une fois par mois j'attrapais une blennorragie, généralement rectale, parfois pénienne, une fois dans la gorge (si enflammée que j'avais du mal à avaler, mon seul symptôme). [...] Essayons le Bactrim. Il n'y a pas de danger qu'il perde son efficacité si j'en prends trop longtemps? Non. Ça n'est pas toi qui deviens résistant au médicament, mais elle - la population entière des bactéries - qui peut muter et devenir résistante à l'antibiotique, mais si ça arrive, tout le monde augmentera la dose et puis c'est tout. » (White, La symphonie des adieux, 1998, p. 98-99).

97. Cyr, Journal intime d'Éric, séropositif, 2001, p. 32-33. 
années d'utilisation chez une personne vivant avec le VIH/sida (PVVIH) impliquée dans une relation stable ${ }^{98}$ :

La capote [...] protège du sexe. Et elle y parvient si bien, que, comme chacun sait, [...] elle empêche toute pénétration assez langoureusement digne de ce nom (on débande, on ne sent rien). C'est la raison pour laquelle la capote n'est, n'a été, ne sera jamais utilisée que dans des circonstances exceptionnelles, et n'est, n'a été, ne pourra jamais être la règle en matière de sexualité humaine. ${ }^{99}$

Le préservatif est de ce fait rejeté, malgré les risques, pour que la PVVIH puisse se réapproprier des sensations érotiques normales, mais sans que le partenaire n'ait donné toujours son accord à une activité risquée de ce type ${ }^{100}$, des situations dont on retrouve plusieurs exemples ${ }^{101},{ }^{102}$ pouvant aller jusqu'à la transmission volontaire du virus par vengeance ou l'acceptation de relations anales passives sans préservatif dans le but de plaire à un partenaire, malgré les préoccupations face à ce type de pratiques dont les risques sont connus ${ }^{103}$. Les possibilités que le préservatif puisse se briser ou glisser et contribuer ainsi à l'infection sont aussi envisagées, démontrant par là les limites de ce mode de protection ${ }^{104,105}$.

Dans d'autres situations, les risques peuvent aussi être assumés en modulant l'usage du préservatif en fonction du statut infectieux des partenaires, du positionnement actif ou

98. « Il y a quatre ans que maintenant il baisait sans capotes avec son mec parce qu'il en avait marre. » (Dustan, Je sors ce soir, 1997, p.28).

99. Dustan, Génie divin, 2001, p. 123-124.

100. « J'ai débandé comme d'habitude dès que j’ai eu mis la capote. Il était de dos. J'ai retiré la capote [...] et ça m'a fait rebander de penser que je pouvais le baiser comme ça, il ne s'était aperçu de rien.[...] Je l'ai baisé en sentant tout. Je me suis retiré pour gicler, c'était tellement bon d'avoir un orgasme comme ça, comme avant quand j'étais vivant. » (Dustan, Plus fort que moi, 1998, p. 70).

101. « Au lit je lui ai mis une capote [...] et trente secondes plus tard le compte était bon. Je me suis retourné et là j'ai vu la capote sur le bord du lit, sans rien dedans, et j'ai dit T'as retiré la capote ?, et il a dit Oui, j'aime pas ça, et je suis devenu vert. » (Dustan, Plus fort que moi, 1998, p. 61-62).

102. «Mais pourquoi n'as-tu pas insisté pour que je porte la capote ? demande le Malien [...] Et toi, tu m'as bien fourré sans protection, ajoute le Malien [...] Alors, toi, tu t'en fous de passer des choses aux autres, hein ? Tu t'en fous d'infecter un Nègre, hein ? [...] J'étais sain avant de venir au Canada. C'est un Blanc qui m'a passé ça. Ce sont les Blancs qui ont passé ça aux Noirs. C'est bien le moins que tu paies. » (Chabot, Innocence, 2007, p. 108-109).

103. «Le jeune s'inquiète, car il a déjà entendu parler des risques des relations sexuelles sans protection ; mais le Malien continue à s'enfoncer en ignorant ses objections et ses plaintes.[...] Imonfri est bâti comme un athlète. Il doit certainement être en santé. De toute façon, il ne lui passerait pas une maladie, se convainc-il. [...] Le jeune est prêt à tout pour lui plaire. » (Chabot, Innocence, 2007, p. 145).

104. « Je ne pense pas à Vincent avec qui la capote a claqué l'année dernière, il y avait du sang, et trois mois après il était séropositif. » (Dustan, Je sors ce soir, 1997, p. 88).

105. «Peu de temps après, Jean-François perdait sa capote dans mon cul. En décembre j'ai fait une rétinite. » (Dustan, Plus fort que moi, 1998, p. 47). 
passif dans la relation anale et de la maîtrise de l'éjaculation ${ }^{106}$. L'abandon du préservatif peut aussi obéir à une décision prise en pleine conscience après discussion entre les partenaires sérodiscordants pour signifier la profondeur du lien de couple ${ }^{107}$.

Les romans analysés renvoient à des représentations contrastées de l'épidémie du VIH/ sida et à ses répercussions sur la vie sexuelle et la prévention. Cette dernière obéit à différents modèles qui mettent en évidence les possibles configurations dans ce domaine et la variété des stratégies dont certaines assument délibérément la prise de risques de transmission du VIH. On peut y voir comme le souligne Pivert (2009 : 19) une perspective nouvelle dans la littérature homosexuelle qui, grâce à des auteurs comme Rémès (2003) est « [...] doublement subversive, dans la mesure où elle va à l'encontre des repères moraux de la société mais aussi d'une partie de la communauté homosexuelle elle-même ».

\section{Conclusion}

La transmission des valeurs, des normes et des comportements quant à la sexualité, fait appel à de nombreuses modalités selon les cultures. Alors qu'elle se réalise à travers des apprentissages corporels et des traditions orales dans les sociétés dites " traditionnelles ", dans les sociétés avec écriture et imprimerie, elle se fait aussi à travers la publication de manuels érotiques et de romans. Les textes romanesques, tout en proposant des scénarios sexuels conformes aux normes sociales, mais aussi transgressifs, amènent les lecteurs, à travers l'imaginaire, à bâtir un univers affectif et érotique qui peut les orienter dans l'expression de leur sexualité. Ceux-ci peuvent y trouver une confirmation de leurs propres expériences ou une ouverture sur de nouvelles possibilités d'expression, en offrant non seulement un registre de pratiques sexuelles qui peut alimenter les scénarios interpersonnels et intrapsychiques (Simon et Gagnon 2005 ; Gagnon 2008).

De ce point de vue, les romans que nous avons analysés, et dont la thématique traite $\mathrm{du} \mathrm{VIH} /$ sida dans une optique essentiellement homosexuelle, offrent des perspectives sur les constructions de l'espace urbain et de ses territoires de sociabilité, sur les choix des orientations sexuelles (Bozon 2001), sur les modulations affectives et sensorielles liées à l'expression de la sexualité. Ils permettent aussi d'établir un registre des pratiques sexuelles, qui vont des préliminaires jusqu'aux paraphilies, tout en situant les motivations et les affects complexes qui les accompagnent. Ces romans enfin présentent des scénarios préventifs face à la transmission du VIH/sida qui correspondent aux normes de prévention ou, au contraire, s'en éloignent de façon délibérée quand, parfois, des situations sentimentales ou de relations de pouvoir entre les partenaires rendent malaisée la protection.

106. «J'ai réfléchi [...] Je préfère baiser sans capote. Je prends le risque. [...] Les jours suivants, on a donc baisé sans capote. [...] Je ne jouissais jamais en lui. Comme je ne mouille pas, le risque était faible. Il voulait gérer le virus comme ça. C'était son choix, sa liberté, comme il disait. Lui, par contre, me remplissait. Plusieurs fois par jour même. [...] Ce n'était pas la première fois que j'étais en couple avec un séronég et que j'agissais de la sorte : me faire enculer sans capote et laisser jouir dedans. Enculer sans capote mais ne pas jouir. » (Rémès, Serial fucker : journal d'un barebacker, 2003, p. 181).

107. « [...] il m’a regardé droit dans les yeux. - Ça suffit ! Ce n'est pas à toi de me protéger. Je suis assez grand pour m'en charger tout seul. [...] Maintenant, tu vas t'abandonner. Et me permettre de t'aimer, O.K. ? [...] J'ai pris une grande, une très grande respiration. Et j'ai lâché prise. Il a fait de moi ce qu'il a voulu : il m'a comblé. » (Cyr, Journal intime d'Éric, séropositif, 2001, p. 74). 
Les représentations du $\mathrm{VIH} /$ sida tendent aussi à se diversifier, se banalisant suite aux progrès médicaux, tout en continuant, par la prise de risques que le virus fait exister, à constituer une source d'excitation. Les romans contribuent, même si leur traitement narratif tend à privilégier des descriptions plutôt stéréotypées des pratiques sexuelles, au dispositif de sexualité proposé par Foucault en insistant sur « les sensations du corps, la qualité des plaisirs, la nature des impressions aussi ténues et imperceptibles qu'elles soient » (1976 : 140-141), et en portant sur les tout petits liens sociaux, ici érotiques, dont Laplantine (2003) a montré l'intérêt anthropologique. L'intérêt de ces romans pourrait se situer au plan des dilemmes affectifs et éthiques qu'ils soulèvent et qui peuvent contribuer aux processus de réflexivité dont Giddens (1994) a montré l'importance dans las sociétés modernes.

Toutefois, les romans entrent en compétition avec d'autres sources d'imaginaire et de réflexivité que sont par exemple les films et les séries télévisées, mais aussi avec internet, formant un vaste ensemble de récits sexuels (sexual stories) définis comme des « narrations de la vie intime, axées principalement sur l'érotique, le genre et le relationnel » (Plummer 1995 : 6), des récits qui occupent de plus en plus l'espace public et qui contribuent à la fois à problématiser la sexualité de multiples façons et à rendre plus délicates les distinctions entre fantasmes et réalités. Il reste cependant à analyser la réception de ces différents matériaux par les lecteurs afin de pouvoir mieux comprendre leur contribution à la transmission et à l'apprentissage de la sexualité, dont la littérature constituerait un relais dans la société contemporaine.

\section{Romans cités}

Canesi, Michel et Jamil Rahmani, 2006. Le syndrome de Lazare. Monaco : Éditions du Rocher.

Снавот, Denis-Martin, 2007. Innocence. Paris : Éditions Textes gais.

Cyr, Mario,

1997. L'éternité serait-elle un long rêve cochon? Boucherville : Éditions de Mortagne.

2001. Journal intime d'Éric, séropositif. Montréal : Les Intouchables.

Delorme, Pascal, 2001. Afin que personne ne puisse nous faire de mal. Montréal : Stanké.

Deroche, Frank, 2002. Effets secondaires. Paris : Le Dilettante.

Dustan, Guillaume,

1997. Je sors ce soir : roman. Paris : POL.

1998. Plus fort que moi : roman. Paris : POL.

2001. Génie divin. Paris : Balland.

Gendron, Marc, 1997. Le prince des ouaouarons. Montréal : XYZ éditeur.

Henri, Nicolas, 2004. Bleu Caraïbes. Roman. Paris : Cylibris.

Maupin, Amistead, 1997, [1987]. D’un bord à l'autre (traduit par Gwenaël Hubert). Paris : Passage du Marais. [Titre original de l'ensemble : Tales of the city Series. Vol 5 : Significant others. New York : Harper Collins Publishers.]

Py, Olivier, 2002. Paradis de tristesse. Arles : Actes Sud.

RÉMĖs, Éric, 2003. Serial fucker : journal d'un barebacker. Paris : Éditions Blanche. 
White, Edmund,

1998, [1997]. La symphonie des adieux (traduit de l'américain par Marc Cholodenko) Paris : Plon [The Farewell Symphony. New York : Alfred A. Knopf].

2000, [2000]. L'homme marié (traduit de l'américain par Anne Rabinovitch). Paris : Plon [The married man. New York : Knopf].

\section{Références citées}

Achilles, Nancy, 1967. « The development of the homosexual bar as an institution », in John Gagnon et William Simon (éds), Sexual Deviance. New York : Harper et Row, p. 228-244.

ARCAND, Bernard, 1991. Le jaguar et le tamanoir : vers le degré zéro de la pornographie. Montréal : Éditions le Boréal.

Alexandrian, Sarane, 1995. Histoire de la littérature érotique. Paris : Payot.

Bataille, Georges, 1957. L'érotisme. Paris : Minuit.

BeCH, Henning, 1999. «Representing Lust in public », in Mike Featherstone (éd.), Love and Eroticism. Londres : Sage Publications, p. 215-242.

Blacking, John, 1985. « Movement, Dance, Music and the Venda Girls’ Initiation Cycle », in Paul Spencer (éd.), Society and the Dance. Cambridge : Cambridge University Press, p. 64-91.

Bolton, Ralph, 1992. «Mapping Terra Incognita : Sex Research for Aids Prevention - An Urgent Agenda for the 1990s ", in Gilbert Herdt et Shirley Lindenbaum (éds), The Time of Aids : Social Analysis, Theory, and Method. Londres : Sage Publications, p. 124-158.

BoundiBA, Abdelwahab, 2003. La sexualité en Islam. Paris : PUF.

Bourdier, Frédéric, 2001. Sexualité et sociabilité en Inde du Sud. Familles en péril au temps du sida. Paris : Éditions Karthala.

Bourneuf, Roland, 2007. Pierres de touche. Essai. Québec : L'instant même.

Bozon, Michel, 2001. «Orientations intimes et constructions de soi. Pluralité et divergences dans les expressions de la sexualité, Sociétés contemporaines, 1 (41-42), p. 11-40.

Brulotte, Gaétan, 1998. Euvres de chair. Figures du discours érotique. Québec : Presses de l'Université Laval.

Bruner, Edward M., 1956. « Cultural Transmission and Cultural Change », Journal of Anthropology, 12 (2), p. 191-199.

CAILlois, Roger, 1958. Les jeux et les hommes. Paris : Gallimard.

Coldins, Alan (éd.), 2007. Cities of Pleasure. Sex and the Urban Socialscape. New York : Routledge.

Connaton, Michel, 1990. Pouvoir et sexualité dans le roman africain. Paris : L'Harmattan.

Deligne, Chloé, Koessan Gabiam, Mathieu Van Criekingen et Jean-Michel Decroly, 2006. « Les territoires de l'homosexualité à Bruxelles : visibles et invisibles », Cahiers de géographie du Québec, 50 (140), p. 135-150.

Demczuk, Irène et Frank Remiggi (éds), 1998. Sortir de l'ombre : histoires des communautés lesbienne et gaie de Montréal. Montréal : VLB éditeur.

De Singly, François, 1993. « Le livre et la construction de l'identité », in François de Singly (éd.), Identité, Lecture, Écriture. Paris : B.P.I., p. 131-152.

Evola, Julius, 1968. Métaphysique du sexe. Paris : Payot.

Évrard, Frank, 2003. La littérature érotique ou l'écriture du plaisir. Toulouse : Milan.

Foucault, Michel, 1976. Histoire de la sexualité. Tome 1. La volonté de savoir. Paris : Gallimard. 
Gagnon, John, 2008. Les Scripts de la sexualité. Essais sur les origines culturelles du désir. Paris : Payot.

GARNIER, Xavier, 1999. La magie dans le roman africain. Paris : PUF.

Giddens, Anthony, 1994. Les Conséquences de la modernité. Paris : L'Harmattan.

Gilles, Audrey, 2009. "La poésie du phallus dans Le Condamné à mort de Jean Genet », @nalyses [En ligne], Articles courants, 20 e siècle, mis à jour le : 26/10/2009, <http://www.revue-analyses.org/index. php?id=671>. Page consultée le 3 avril 2010.

Goody, Jack, 2003. La peur des représentations. L'ambivalence à l'égard des images, du théâtre, de la fiction, des reliques et de la sexualité. Paris : La Découverte.

Harry, Joseph, 1974. « Urbanization and the Gay Life », The Journal of Sex Research, 10 (3), p. 238-247.

Hervieu-LÉger, Danièle, 1997. « La transmission religieuse en modernité », Social Compass, 44 (1), p. 131-143.

Hubier, Sébastien, 2007. «"L'école des filles". Le Bildungsroman érotique de Fanny Hill à Emmanuelle », in Philippe Chardin (éd.), Roman de formation, roman d'éducation dans la littérature française et dans les littératures étrangères. Paris : Kimé.

Hubin, Alexandra, Pascal De Sutter et Christine Reynaert, 2008. «L'utilisation de textes érotiques dans l'éveil du désir sexuel féminin », Réalités en gynéco-obstétrique, 134, Novembre/Décembre. <www.performancesmedicales.com/gyneco/Encours/.../09.pdf >.

Jodelet, Denise, 1989. Les représentations sociales, Paris : PUF.

Kashamura, Anicet, 1973. Famille, sexualité et culture. Essai sur les mours sexuelles et les cultures des peuples des Grands Lacs africains. Paris : Payot.

Kraakman, Dorelies, 1994. « Reading Pornography Anew : A Critical History of Sexual Knowledge of Girls in French Erotic Fiction, 1750-1840 », Journal of the History of Sexuality, 4 (4), p. 517-548.

KrIGE, E.J., 1968. « Girls’ Puberty Songs and their Relation to Fertility, Health, Morality and Religion among the Zulu », Africa, 38, p. 173-98.

Lallemand, Suzanne, 1985. L'apprentissage de la sexualité dans les contes de l'Afrique de l'Ouest. Paris : L'Harmattan.

LAPLANTine, François,

1986. Anthropologie de la maladie : étude ethnologique des systèmes de représentations étiologiques et thérapeutiques dans la société occidentale contemporaine. Paris : Payot.

1997. Le métissage. Paris : Flammarion.

2003. De tous petits liens. Paris : Mille et une nuits.

Le Breton, David,

1991. Passions du risque. Paris : Métailié.

2006. La saveur du monde. Une anthropologie des sens. Paris : Métailié.

LÉvy, Joseph Josy et Alexis Nouss, 1994. Sida-fiction. Essai d'anthropologie romanesque. Lyon : Presses de l'Université de Lyon.

LÉvy, Joseph Josy,

1996. « Des jeux érotiques au temps du sida et de leurs enjeux éthiques », Cahier de recherche éthique, 19, p. 227-236.

2006. « Drogues et sexualité », Drogues, Santé et Société, 5 (2), p. 11-48.

Maring, Joel M. et Lilliane E. Maring, 1997. « Japanese Erotic Folksong : From Shunka to Karaoké », Asian music, 28 (2), p. 27-49. 
Marshall, Donald S., 1971. « Sexual Behavior on Mangaia », in Donald S. Marshall et Robert C. Suggs (éds), Human Sexual Behavior: Variations in the Ethnographic Spectrum. New York: Basic Books, p. 103-162.

Mauss, Marcel, 1936. «Les techniques du corps », Journal de Psychologie, 32 (3-4), p. 365-372.

Médico-Vergriete, Denise et Joseph Josy Lévy, 1999. Le baiser. Montréal : Éditions Stanké.

Melody, Michael E. et Linda M. Peterson, 1999. Teaching America About Sex : Marriage Guides and Sex Manuals from the Late Victorians to Dr. Ruth. New York : New York University Press.

Messenger, John C., 1971. «Sex and Repression in an Irish Folk Community », in Donald S. Marshall et Robert C. Suggs (éds), Human Sexual Behavior : Variations in the Ethnographic Spectrum. New York : Basic Books, p. 3-37.

Murphy, Timothy F. et Suzanne PoIrier (éds), 1993. Writing AIDS : Gay Literature. Language, and Analysis. New York : Columbia University Press.

NiCOLAs, Lucienne, 2002. Espaces urbains dans le roman de la diaspora haïtienne. Paris : L'Harmattan.

Ohmagari, Kayo et Fikret Berkes, 2004. « Transmission of Indigenous Knowledge and Bush Skills Among the Western James Bay Cree Women of Subarctic Canada », Human Ecology, 25 (2), p. 197-222.

Paxton, Susan, 2002. « The Paradox of Public HIV Disclosure », AIDS Care, 14 (4), p. 559-567.

PARKer, Richard, 2001. « Sexuality, Culture and Power in HIV/AIDS Research », Annual Review of Anthropology, 30, p. $163-179$.

Pelissier, Catherine, 1991. « The Anthropology of Teaching and Learning », Annual Review of Anthropology, 20 , p. $75-95$.

Pivert, Benoît, 2009. « Préface », in Benoît Pivert (éd.), Homosexualité(s) et littérature, Cahiers de la Revue d'Art et de Littérature, Musique (RAL,M), p. 11-34.

Plummer, K. (1995). Telling sexual stories: Power, change, and social worlds. London : Routledge.

Ranke-Heinemann, Uta, 1990. Des eunuques pour le royaume des cieux : L'Église catholique et la sexualité. Paris : Laffont.

ReIss, Ira, (1986). Journey into sexuality. An Exploratory Voyage. Englewod Cliffs, NJ : Prentice-Hall.

Rivière, Claude, 1995. Les rites profanes. Paris : PUF.

Sibalis, Michael, 2004. «Urban Space and Homosexuality : The Example of the Marais, Paris' 'Gay Ghetto'», Urban Studies, 41 (9), p. 1739-1758.

Simon, William et John Gagnon, 2005. «Sexual Scripts : Permanence and Change », Archives of Sexual Behavior, 15 (2), p. 97-120.

Soulié, Bernard, 1993. L'art d'aimer au Japon. Paris : Solar.

Stefaniszyn, Bronislaw, 1964. Social and Ritual Life of the Ambo of Northern Rodhesia. Londres : Oxford University Press.

Suggs, Robert C., 1966. Marquesan Sexual Behavior. New York : Harcourt, Brace and World.

Turner, James, G., 2003. Schooling Sex : Libertine Literature and Erotic Education in Italy, France and England 1534-1685. Londres et New York : Oxford University Press.

TruebA, Henry T., 1993. « The Dynamics of Cultural Transmission », in Henry T. Trueba, Cirenio Rodriguez, Yali Zou et José Cintron (éds), Healing Multicultural America. Londres : The Falmer Press, p. 10-28.

VAN Gulik, Robert, 1977. La vie sexuelle dans la Chine ancienne. Paris : Gallimard.

Volet, Jean-Marie, Hélène Jaccomard et Phillip Winn, 2002. « La littérature du sida : genèse d'un corpus », The French Review, 75 (3), p. 528-541. 
Weitman, Sasha, 1999. « On the Elementary Forms of Socioerotic Life », in Mike Featherstone (éd.), Love and Eroticism. Londres : Sage publications, p. 71-110.

White, Charles, M.N., 1953. « Conservatism and Modern Adaptation in Luvale Female Puberty Ritual », Africa, 23 (1), p. 15-23.

Wouters, Cas, 1999. "Balancing Sex and Love since the 1960s Sexual Revolution », in Mike Featherstone (éd.), Love and Eroticism. Londres : Sage, p. 187-214.

Zabus, Chantal, 2007. Between Rites and Rights : Excision in Women's Experiential Texts and Human Contexts. Stanford : Stanford University Press. 